\title{
Congestion Control Techniques in WSNs: A Review
}

\author{
Babar Nawaz ${ }^{1}$, Khalid Mahmood ${ }^{*}$, , Jahangir Khan ${ }^{3}$, Mahmood ul Hassan ${ }^{4}$, Ansar Munir Shah ${ }^{5}$, \\ Muhammad Kashif Saeed ${ }^{6}$
}

\begin{abstract}
Department of Computer Science, IIC University of Technology, Phnom Penh, Kingdom of Cambodia 1, 2, 3,4,5,6 Department of Computer Science, University College of Alwajh, University of Tabuk, Kingdom of Saudi Arabia ${ }^{4}$ Department of Computer Science \& IT, Institute of Southern Punjab (ISP) Multan, Pakistan ${ }^{5}$
\end{abstract}

\begin{abstract}
Congestion control has a great importance in wireless sensor network (WSN), where efficient application of congestion control mechanisms can prolong the network lifetime. Thus, proper examination is needed to improve more refine way to address the congestion occurrence and resolution. While designing congestion control techniques, the maximum output can be achieved by efficient utilization of required resources within WSN. From last few years several approaches have been brought in, that consist of routing protocols which provide support with congestion control, congestion prevention, and reliable data routing. In old schemes the topology reset and extent traffic drop take place because sink node executes the congestion avoidance. Therefore, node level congestion avoidance, detection, congestion preventing, and resolution mechanisms have been proposed during past few years. Our paper provides a brief overview and performance comparison of centralized and distributed congestion control algorithms in WSN.
\end{abstract}

Keywords-WSNs; congestion control; congestion preventing; reliable data

\section{INTRODUCTION}

Wireless sensor network (WSN) consists of very small wireless devices deployed in a huge geographical area to examine the surrounding environment. Through, multi-hop routing protocols the oversee information is transmitted from sensor to sensor towards sink. Information collection and examination takes place at sink node. The sensor nodes have limited power, energy, and communication resources [1] [2] [3]. Different routing schemes are used to efficiently handle the WSN's resources in order to achieve the better performance. Congestion control is the prominent area for the researchers as network traffic is increasing rapidly with frequent changes in buffering mechanisms [5].

The main task of WSNs is to provide transport and network protocols functionalities for reliable data transfer over the unreliable channels and nodes and also deals with fault tolerance [7]. Usually for network level, the cause of node failure is due to the changes in path and topology that must be treated properly to reduce the relative packet loss and energy exhaustion [8]. At transport level, congestion should be managed to prevent the data loss by fair distribution of the bandwidth for all the network nodes especially for the distant nodes [8].

Congestion control helps in avoiding extent traffic drop that is why congestion control is of demanding concern. Congestion control comprises of three phases. The first phase is congestion detection in which congestion is detected at sensor nodes while in notification phase; a problematic sensor node has been notified of congestion after the detection of congestion. Lastly, in congestion mitigation phase; the congestion is checked and suitable data rate is applied. There are three stages for congestion control; congestion detection, prevention, and congestion control. While designing WSNs, the main focus should be on congestion control to carry out the maximum life of network by efficient use of the resources [1].

Current congestion control approaches have some difficulties, such as the onward traffic management does not consider the traffic estimation on the substitute paths [4]. From source sensor node to sink, the priority is set on hop count instead of actual packet delay [4-6]. Moreover, the distribution of traffic loads at congested and substitute paths are not handled properly.

This survey paper provides a brief overview on congestion control mechanisms by graphically illustrating the working of some of the congestion control algorithms. Moreover, we have provided the performance comparison by examining the parameters mentioned in each of the congestion control algorithm discussed in this survey paper. Moreover, this paper also highlights the shortcomings of the existing congestion control mechanisms.

\section{LITERATURE REVIEW}

Congestion control algorithms for WSN are extensively discussed in recent past years [8] [9]. In WSNs, congestion occurs at two levels i-e node and link level congestion. Fig. 1 illustrates the congestion levels in WSN.

Congestion occurs when the amount of received data at particular node is higher than its transmitted data which subsequently causes the drop of packets. Generally, congestion occurs at the nodes that are very close to the sink node.

Node-level congestion badly affects the performance of the affected node in WSN. It causes the loss of energy due to the higher packet loss ratio and consequently disconnects the affected node from the network causing certain route unavailability. Energy depletion and poor routing have negative impact on the performance of the network and badly reduce the overall reliability and lifespan of the network.

\footnotetext{
*Corresponding Author.
} 


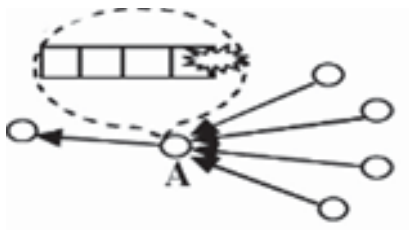

(a) Node Level

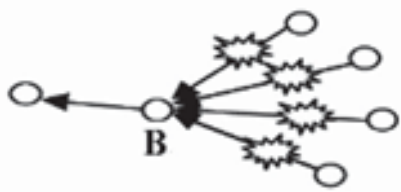

(b) Link Level
Fig. 1. Congestion Level in WSNs.

Collision, competition and bit error are the reasons for link level congestion occurrence. In Fig. 1 node level congestion occurs at node A. A suitable congestion control scheme can efficiently manage the transmission of data in order to prevent from loss of energy or it can change the route of surplus packets. It will increase the sink node output and frequently assists the WSN application by efficiently monitoring the environment. The minimum packet loss also increases the reliability of the WSN application. Therefore, preventing routing holes, frequently and on time data delivery will increase the life span of the network.

Link level congestion occurs at node B in Fig. 1 where node $B$ receives few packets despite its neighbor sends with full data rate. The reliability of the WSN is badly affected when sink receives fewer packets. In such kind of situation MAC layer should be managed by a congestion control algorithm that helps coordinates to access the medium and prevents form collision.

Mainly, congestion control algorithms are distributed in three types namely congestion mitigation, congestion detection, and reliable data transmission algorithms as shown in Fig. 2. Congestion mitigation algorithms are reactive in nature which react and control the congestion whenever network suffers from congestion. Mostly, these algorithms are working with MAC and network layer operations, and in few circumstances transport layer operations are performed by them.

Congestion detection algorithms are employed to prevent the network from congestion occurrence. MAC and network layer operations are usually handled by such kind of algorithms.

Reliable data transmission algorithms are used to control the congestion in a network in such a way that these algorithms try to get back all the lost information or some part of it. Usually, these algorithms are used at the time when whole information is necessary for application. The transport layer approaches are involved in these algorithms.

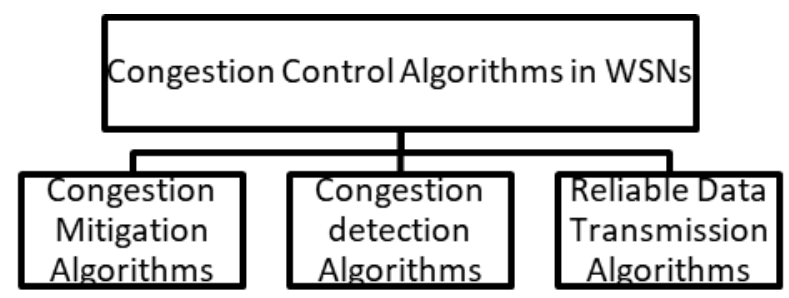

Fig. 2. Congestion Control Algorithms.

\section{CONGESTION CONTROL TeChNIQUeS}

\section{A. RCRT}

RCRT is a transport protocol that determines and allocates the data rate to resolve the congestion [7]. The sink node performs congestion detection, rate control, and allocation. This scheme has some limitations like, the slow convergence rate and fails to figure out the flow constrained in congestion area. The operational scheme of RCRT is illustrated in Fig. 3.

\section{B. $I 2 M R$}

I $2 \mathrm{MR}$ is a routing protocol aided with the congestion control mechanism [8]. To remove congestion, the I2MR protocol keeps multiple substitute paths for routing information. Experimental weighted moving averages are used to detect the congestion by spotting source node's single buffer. The protocol informs the source node to decrease the transmission rate for controlling congestion. The transmission rate is decreased by the source node through redirecting the traffic to the substitute routes. The I2MR protocol has some restrictions like a massive data loss is unavoidable if the substitute's routes are not available. The rate balancing comprises on one-fourth, one-sixth or one-eighth of the data link rate instead of comprising the predicted traffic that consequence the faulty channel.

\section{TADR}

The TADR protocol illustrates a hybrid scalar potential area that consists of queue length and depth area [8]. Initially TADR routes the packets to the sink from the shortest paths. Later on the functionality of traffic awareness is developed in it. If congestion occurs, TADR sends the packets to the substitute route that comprises of less-loaded or idle nodes. To prevent hot spots, a bypassing hot-spot rule is brought in. The main drawback of TADR is to find out the time variant potential area that leads to the traffic diversion [8]. The operations of TADR algorithm are shown in Fig. 4.

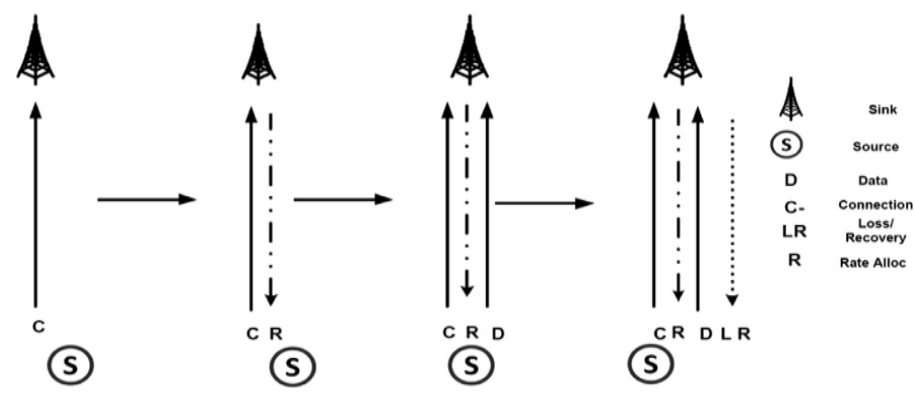

Fig. 3. RCRT Setup Phase [6]. 


\section{Buffer-Based Congestion Avoidance Scheme}

Buffer-based congestion avoidance scheme is checked against several MAC protocols, like TDMA with unchanged scheduling and CSMA with implicit ACKs, as illustrated in Fig. 5 [10]. A 1/k buffer solution addresses the hidden terminal problem. In this scheme the fairness for load balancing and buffer access on various routes is assured. The scheme is illustrated in Fig. 5.

\section{E. DAIPaS}

The DAIPaS protocol detects the congestion by including the channel interference, buffer occupancy and residual energy of a single node [11]. The nature of selection of shortest routes for traffic is dynamic that prevent it from the congested nodes. In setup phase, DAIPaS broadcasts a HELLO beacon in the vicinity of the network by setting the head nodes level ID to 0 . When nodes receive the HELLO message, they further broadcast the message by increasing the ID value by 1 . This method continues as far as a unique level ID is assigned to each node in the network. Each node maintains an ID to discover substitute paths or manipulate shortest path towards the sink. The shortest path is determined by examining the flow from the highest to the lowest value. The comparative analysis between incoming and transmission flow along with buffer occupancy determines the congestion. The packet sequence number in the receiving node's header is set to FALSE when DAlPaS goes into the soft stage and the packet is transmitted to the further node. The sending selects the substitute paths on receiving the value and will carry on sending the data on it. The DAlPaS protocol sets the minimum threshold for buffer occupancy and it moves into the hard phase if the buffer size surpasses the minimum threshold or the inward data flow exceed the transmission rate. Consequently, DAIPaS redirects the packets on new route. The DAlPaS rearranges the topology by eliminating the node from the current route.

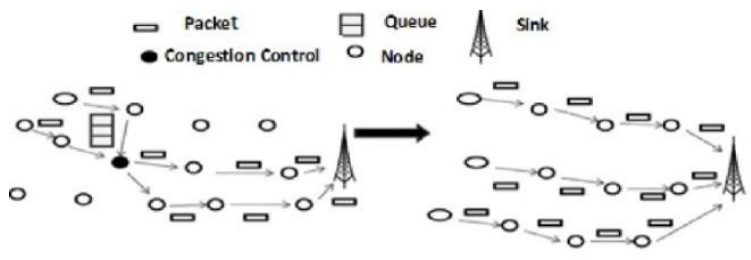

(a)

(b)

Fig. 4. Illustration of TADR Operations.

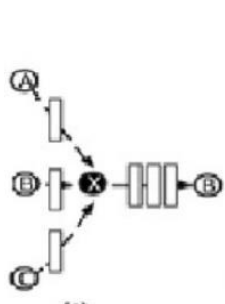

(a)

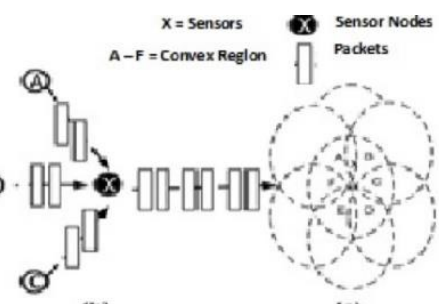

(b) (c)
Fig. 5. Illustration of Buffer-Based Congestion Avoidance Scheme.

\section{F. Fusion}

Fusion is a method for checking the congestion on queue length [12]. Additionally, to handle the congestion, fusion relies on hop-by-hop flow control, prioritized MAC approaches and rate control. Insufficient buffer spaces occur whenever packets are dropped downstream and packet transmission is stopped. During the comparison with other non-congested sensor nodes, it is noticed that this priority is managed by congested sensor nodes through reducing random back-off timer. Lack of balance in data transfer happen towards the nodes that are situated far from the sink node. To prevent this problem, the rate limitation metrics of traffic are accepted. A prioritized CSMA-based MAC manages congestion in congested sensors by reducing random back-off timer. Accordingly, fusion optimizes fairness and maintains effective output.

\section{G. WRCP (Wireless Rate Control Protocol)}

WRCP (Wireless Rate Control Protocol) is a Wireless Sensor Network protocol that is used to improve convergence time of rate control [13]. It is designed by using a receiver capacity model that is a novel interference model. This model enables each receiver to find out the accurate available capacity which is used by WRCP to get a fair rate allocation. WRCP shares this capacity information in between competing flows in a neighborhood. By using explicit capacity information, WRCP shows fast convergence time that result in small end-to-end delays.

\section{H. TRCCIT (Tunable Reliability with Congestion Control for Information Transport)}

TRCCIT protocol ensures the appropriate reliability level of the hybrid acknowledgement (HACK) approach [14]. The forwarder overhears the retransmission of sender's packets by setting a hop-by-hop control.

In the situation when the required reliability level is beforehand achieved, the receiver depresses the received packet and sends a simple acknowledgment to the sender to prevent from packet retransmission after the completion of timeout. At the time of congestion detection the TRCCIT addresses to control congestion through multipath forwarding. But utilization of multiple paths forwarding is not continually possible and accordingly TRCCIT congestion control approach is not sufficient.

\section{I. $\quad$ DPCC (Decentralized Predictive Congestion Control)}

DPCC is a WSN protocol that comprises an adaptive flow and back-off interval selection approaches that get the job through distributed power control (DPC) and energy efficiency [15]. Initially, DPCC detects the congestion with the help of queue utilization and the embedded channel quality. The adaptive back off interval selection approach applies a rate whereas the adoptive flow control approach selects that suitable rate. Fig. 6 shows the rate selection process. To assure the weight fairness during congestion, the associated weight of 
each packet is updated by an optional scheduling approach. Simulation outcome shows that the DPCC increases performance and decreases the congestion through congestion detection and congestion prevention process.

\section{J. GMCAR (Grid-based Multipath with Congestion Avoidance Routing)}

GMCAR protocol is an effective QoS routing protocol used in gridded sensor networks [16]. It uses the concept of splitting the wireless sensor network region into grids. In each grid, one master node is selected from associated sensor nodes. All master nodes from each grid collaborate with each other and also responsible to process and route the data of associated sensor nodes. In the routing table of each master node, multiple diagonal paths as routing entries are stored that link master node to the sink. In case of congestion occurrence, a congestion control approach is suggested to reduce the congested areas. When compared to other QoS protocols, the outcome of simulation shows that GMCAR protocol has the potential to achieve the delay reduced to $24.7 \%$, network output increased by $8.5 \%$ and $19.5 \%$ energy saving. Moreover, it shows superiority in accomplishing better available storage usage.

\section{K. TASA (Traffic Aware Scheduling Algorithm)}

TASA (Traffic Aware Scheduling Algorithm) [17] is based on TSCH behavior and it is a centralized scheduling approach. The TASA build a tree-based schedule at sink that has nodes traffic load information. While using the edge of frequency diversity and resources, TASA provides a better output. In graph theory methods, TASA uses matching and coloring for accomplishing the specified objectives. Fig. 7 shows the working of TASA algorithm.

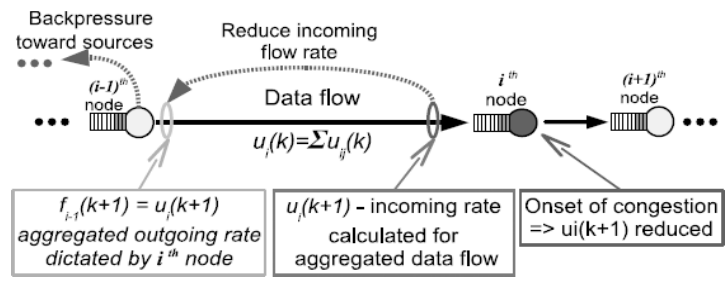

Fig. 6. Rate Selection Overview.
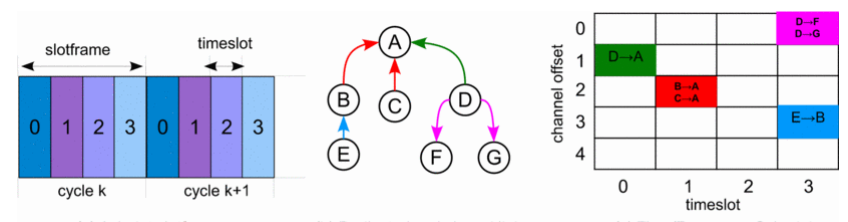

(a) A 4-slot slotframe.

(b) Dedicated and shared links.

Fig. 7. Traffic Aware Scheduling Aware Algorithm.

\section{OTF (On-the-Fly Scheduling)}

OTF (On-the-Fly Scheduling) presents a distributed schedule approach. This approach uses slots to prevent interferences and ensures reliability [18]. As per the network need, OTF adjusts the slot number of the nodes with respect to the traffic load. For schedule adaptation, the resources that are added or removed are sent to the sub-layer for schedule adoption.

\section{PERFormance COMPARISON}

The performance comparison of aforementioned congestion control schemes is based on their operational strategy, congestion detection criteria, congestion notification, congestion control, priority criteria, and control patterns. The comparison is shown in Table I.

TABLE I. CONGESTION CONTROL PROTOCOLS COMPARISON

\begin{tabular}{|c|c|c|c|c|c|c|c|}
\hline \multirow[b]{2}{*}{ References } & \multicolumn{7}{|c|}{ Congestion Routing Protocols } \\
\hline & Protocols & Operational scheme & $\begin{array}{l}\text { Congestion detection } \\
\text { models }\end{array}$ & $\begin{array}{l}\text { Congestion } \\
\text { notification }\end{array}$ & $\begin{array}{l}\text { Congestion } \\
\text { control }\end{array}$ & $\begin{array}{l}\text { Priority } \\
\text { criteria }\end{array}$ & Control Patterns \\
\hline [11] & RCRT & $\begin{array}{l}\text { Congestion } \\
\text { detection, rate } \\
\text { adoption and } \\
\text { allocation }\end{array}$ & Buffer Overflow & $\begin{array}{l}\text { New Rate in } \\
\text { NACK } \\
\text { header, or } \\
\text { Feedback } \\
\text { Rate message }\end{array}$ & $\begin{array}{l}\text { AIMED } \\
\text { rate } \\
\text { control }\end{array}$ & NO & End to End \\
\hline [12] & $12 \mathrm{MR}$ & $\begin{array}{l}\text { Routing support } \\
\text { through congestion } \\
\text { control }\end{array}$ & $\begin{array}{l}\text { Buffer occupancy } \\
\text { and exponential } \\
\text { weighted moving } \\
\text { average }\end{array}$ & $\begin{array}{l}\text { Feedback } \\
\text { Massage }\end{array}$ & Rate control & NO & Hope by Hope \\
\hline [13] & TADR & $\begin{array}{l}\text { Routing with } \\
\text { congestion control }\end{array}$ & $\begin{array}{l}\text { Buffer and Rate } \\
\text { hybrid scalar } \\
\text { positional field }\end{array}$ & & $\begin{array}{l}\text { Resources } \\
\text { control }\end{array}$ & NO & Hope by Hope \\
\hline [14] & $\begin{array}{l}\text { Buffer-based } \\
\text { congestion } \\
\text { avoidance } \\
\text { scheme }\end{array}$ & Congestion control & Buffer Occupancy & $\begin{array}{l}\text { Information in } \\
\text { header }\end{array}$ & $\begin{array}{l}\text { Stop } \\
\text { sending }\end{array}$ & NA & Hope by Hope \\
\hline [15] & DAIPaS & $\begin{array}{l}\text { Dynamic Alternative } \\
\text { path selection }\end{array}$ & $\begin{array}{l}\text { Buffer occupancy } \\
\text { and channel load }\end{array}$ & $\begin{array}{l}\text { Information in } \\
\text { header }\end{array}$ & $\begin{array}{l}\text { Resources } \\
\text { control }\end{array}$ & NO & Hope by Hope \\
\hline
\end{tabular}




\begin{tabular}{|c|c|c|c|c|c|c|c|}
\hline [16] & Fusion & $\begin{array}{l}\text { Flow control, Rate } \\
\text { limiting and } \\
\text { prioritized MAC }\end{array}$ & Buffer and rate & Bit in header & $\begin{array}{l}\text { Stop } \\
\text { sending } \\
\text { prioritized } \\
\text { MAC }\end{array}$ & NA & Hope by Hope \\
\hline [17] & WRCP & $\begin{array}{l}\text { Helps in the network } \\
\text { to conclude the } \\
\text { accurate available } \\
\text { capacity at each } \\
\text { receiver }\end{array}$ & NO & $\begin{array}{l}\text { Information } \\
\text { header }\end{array}$ & $\begin{array}{l}\text { Rate } \\
\text { control }\end{array}$ & NO & Hope by Hope \\
\hline [18] & TRCCIT & $\begin{array}{l}\text { Hop-by-hop control } \\
\text { to overhear the } \\
\text { retransmission of } \\
\text { packets }\end{array}$ & $\begin{array}{l}\text { When congestion } \\
\text { detection the } \\
\text { TRCCIT address to } \\
\text { control congestion } \\
\text { through multipath } \\
\text { forwarding }\end{array}$ & $\begin{array}{l}\text { Send ACK to } \\
\text { sender }\end{array}$ & $\begin{array}{l}\text { Traffic } \\
\text { control }\end{array}$ & NO & Hope by Hope \\
\hline [19] & DPCC & $\begin{array}{l}\text { Dynamic and fair } \\
\text { management of } \\
\text { traffic broadcast. }\end{array}$ & $\begin{array}{l}\text { Queue Utilization } \\
\text { and Channel Quality }\end{array}$ & $\begin{array}{l}\text { Information in } \\
\text { ACK header }\end{array}$ & $\begin{array}{l}\text { Rate } \\
\text { control } \\
\text { adaptive } \\
\text { back off }\end{array}$ & $\begin{array}{l}\text { Pre- } \\
\text { defined } \\
\text { rules }\end{array}$ & Hope by Hope \\
\hline [20] & GMCAR & $\begin{array}{l}\text { Splitting the sensor } \\
\text { network region into } \\
\text { grid }\end{array}$ & $\begin{array}{l}\text { Use a congestion } \\
\text { control mechanism }\end{array}$ & $\begin{array}{l}\text { Information } \\
\text { header }\end{array}$ & $\begin{array}{l}\text { Resources } \\
\text { control }\end{array}$ & NO & Hope by Hope \\
\hline [21] & TASA & $\begin{array}{l}\text { At sink build a tree- } \\
\text { based schedule }\end{array}$ & $\begin{array}{l}\text { Build tree base } \\
\text { schedule }\end{array}$ & $\begin{array}{l}\text { Average } \\
\text { single hope } \\
\text { delay }\end{array}$ & $\begin{array}{l}\text { Traffic } \\
\text { control }\end{array}$ & NO & Hope by Hope \\
\hline [22] & OTF & $\begin{array}{l}\text { use slots to prevent } \\
\text { interferences and } \\
\text { ensures reliability }\end{array}$ & $\begin{array}{l}\text { As per the network } \\
\text { need OTF adjust the } \\
\text { slot number of the } \\
\text { nodes with respect to } \\
\text { the traffic load }\end{array}$ & $\begin{array}{l}\text { Information } \\
\text { header }\end{array}$ & $\begin{array}{l}\text { Resources } \\
\text { control }\end{array}$ & NO & Hope by Hope \\
\hline
\end{tabular}
research. It is a challenging task to develop congestion control techniques with limited resource. This paper gives an inclusive review on the current congestion control techniques. The aim of all the techniques is to increase the life time of the WSNs by using available limited resources. We compared different metrics for congestion detection and controlling. Fast feedback, high transmission, inexpensiveness, low power consumption, fault tolerance, consistency, wear-ability, and complexity of the WSN are the important areas which are addressed in congestion control schemes.

For the future work, we will propose an energy efficient congestion control technique that will overcome the shortcomings of the aforementioned techniques.

\section{ACKNOWLEDGMENT}

We would like to express our gratitude to IIC University of Technology, Kingdom of Cambodia for providing us good research environment.

\section{REFERENCES}

[1] Omidvar, M., Ayatollahitafti, V., \& Farahmand, M. (2017). A Congestion-Aware Routing Algorithms Based on Traffic Priority in Wireless Sensor Networks. Journal of Soft Computing and Decision Support Systems, 4(1), 1-6.

[2] Khalid Mahmood, Muhammad Amir Khan, Mahmood ul Hassan, Ansar Munir Shah, Shahzad Ali, and Muhammad Kashif Saeed, "Intelligent On-Demand Connectivity Restoration for Wireless Sensor
[3] Randhawa, S., \& Jain, S. (2017). Data Aggregation in Wireless Sensor Networks: Previous Research, Current Status and Future Directions. Wireless Personal Communications, 97(3), 3355-3425.

[4] Gholipour, M., Haghighat, A. T., \& Meybodi, M. R. (2017). Hop - by Hop Congestion Avoidance in wireless sensor networks based on genetic support vector machine. Neurocomputing, 223, 63-76.

[5] Zhu, L., Zhang, Z., \& Xu, C. (2017). Secure data aggregation in wireless sensor networks. In Secure and Privacy-Preserving Data Communication in Internet of Things (pp. 3-31). Springer Singapore.

[6] Chen, H. M., Cui, L., \& Zhou, G. (2017). A Light-Weight Opportunistic Forwarding Protocol with Optimized Preamble Length for Low-DutyCycle Wireless Sensor Networks. Journal of Computer Science and Technology, 32(1), 168-180.

[7] Felemban, E. (2017, August). Quality of Information for Wireless Body Area Networks. In Quality, Reliability, Security and Robustness in Heterogeneous Networks: 12th International Conference, QShine 2016, Seoul, Korea, July 7-8, 2016, Proceedings (Vol. 199, p. 171). Springer. Chicago.

[8] Kafi, M. A., Othman, J. B., \& Badache, N. (2017). A Survey on Reliability Protocols in Wireless Sensor Networks. ACM Computing Surveys (CSUR), 50(2), 31.

[9] Li, X., Li, D., Wan, J., Vasilakos, A. V., Lai, C. F., \& Wang, S. (2017). A review of industrial wireless networks in the context of industry 4.0. Wireless networks, 23(1), 23-41.

[10] Shah, S. A., Nazir, B., \& Khan, I. A. (2016). Congestion control algorithms in wireless sensor networks: Trends and opportunities. Journal of King Saud University-Computer and Information Sciences.

[11] J. Paek, R. Govindan,."RCRT: Rate-Controlled Reliable Transport for Wireless Sensor Networks" in ACM SenSys'07, Sydney, Australia:, Nov. 2007. 
[12] Teo, Jenn-Yue, Ha, Yajun, Tham, Chen-Khong, 2008. Interferenceminimized multipath routing with congestion control in WSN for highrate streaming. IEEE Trans. Mobile Comput. 7 (9).

[13] Ren, Fengyuan, Das, Sajal K., Lin, Chuang, 2011. Traffic-aware dynamic routing to alleviate congestion in WSN. IEEE Trans. Parallel Distrib. Syst. 22 (9).

[14] Chen, Yang, 2006. Congestion avoidance based on lightweight buffer management in sensor networks. IEEE Trans. Parallel Distrib. Syst. 17 (9), 934-946.

[15] Charalambos, Vasosu, 2011. DAlPaS: a performance aware conges- tion control algorithm in WSN. In: Proceeding of the IEEE 18th International Conference on Telecommunications.

[16] Hull, Jamieson, Balakrishnan, 2004. Mitigating congestion in WSN. In: Proceedings of the 5th ACM Conference on Embedded Networked Sensor Systems (SenSys).

[17] SRIDHARAN, A. AND KRISHNAMACHARI, B. 2009. Explicit and precise rate control for wireless sensor networks. In Proceedings of the 7th ACM International Conference on Embedded Networked Sensor Systems (SenSys09).
[18] F. K. Shaikh, A. Khelil, and N. Suri. 2009. AReIT: Adaptable reliable information transport for service availability in wireless sensor networks. In Proceedings of International Conferenceon Wireless Networks (ICWN'09). 75-81.

[19] Heikalabad, S. R., Ghaffari, A., Hadian, M. A., \& Rasouli, H. (2011). DPCC: Dynamic Predictive Congestion Control in wireless sensor networks. IJCSI International Journal of Computer Science Issues, 8(1).

[20] Banimelhem, O., \&Khasawneh, S. (2012). GMCAR: Grid-based multipath with congestion avoidance routing protocol in wireless sensor networks. Ad Hoc Networks, 10, 1346-1361.

[21] M. R. Palattella, N. Accettura, M. Dohler, L. A. Grieco, and G. Boggia. 2012. Traffic aware schedulingalgorithm for reliable low-power multihop IEEE 802.15.4e networks. In Proceedings of the 2012 IEEE23rd International Symposium on Personal, Indoor and Mobile Radio Communications (PIMRC'12).327-332.

[22] M. R. Palattella, T. Watteyne, Q. Wang, K. Muraoka, N. Accettura, D. Dujovne, L. A. Grieco, and T. Engel.2016. On-the-fly bandwidth reservation for $6 \mathrm{TiSCH}$ wireless industrial networks. IEEE Sensors Journal16, 2 (Jan. 2016), 550-560. 